\title{
MILKY WAY
}

\section{Some like it warm}

The vast space between stars is filled with gas - ionized, atomic and molecular that is collectively known as the interstellar medium (ISM). The ionized ISM around $\mathrm{H}$ II regions provides important insight not only on how individual stars form but also on how galaxies evolve. Surveys of the ISM covering large swathes of the Milky Way, while costly, are important, as they can uncover details of the structure and dynamics of this ionized gas that are otherwise inaccessible in external galaxies. Recently released observations of the entire Galactic Plane from a South Korean satellite offer a high-fidelity, sensitive view of the warm, ionized hydrogen component $(6,000-10,000 \mathrm{~K})$ of the ISM within the Milky Way.

The Multi-purpose InfraRed Imaging System (MIRIS) was a compact nearinfrared space telescope that launched in November 2013 as the main payload of the Science and Technology Satellite 3 of the Republic of Korea. After 18 months of observations, the satellite concluded its operation in May 2015. The Galactic Plane (pictured) was observed using narrowband filters centred on the Paschen $\alpha(\mathrm{Pa} \alpha)$ emission line from ionized hydrogen atoms in the ISM. Given their longer wavelength, Pa $\alpha$ photons are less affected by dust and therefore allow a deeper view on areas of the Milky Way that are otherwise obscured: the Galactic Centre and dense star-forming regions. The observations cover the whole $360^{\circ}$ of the plane between Galactic latitudes of $-3^{\circ}$ to $+3^{\circ}$. The high sensitivity (better than $16 \mathrm{AB}$ magnitude)

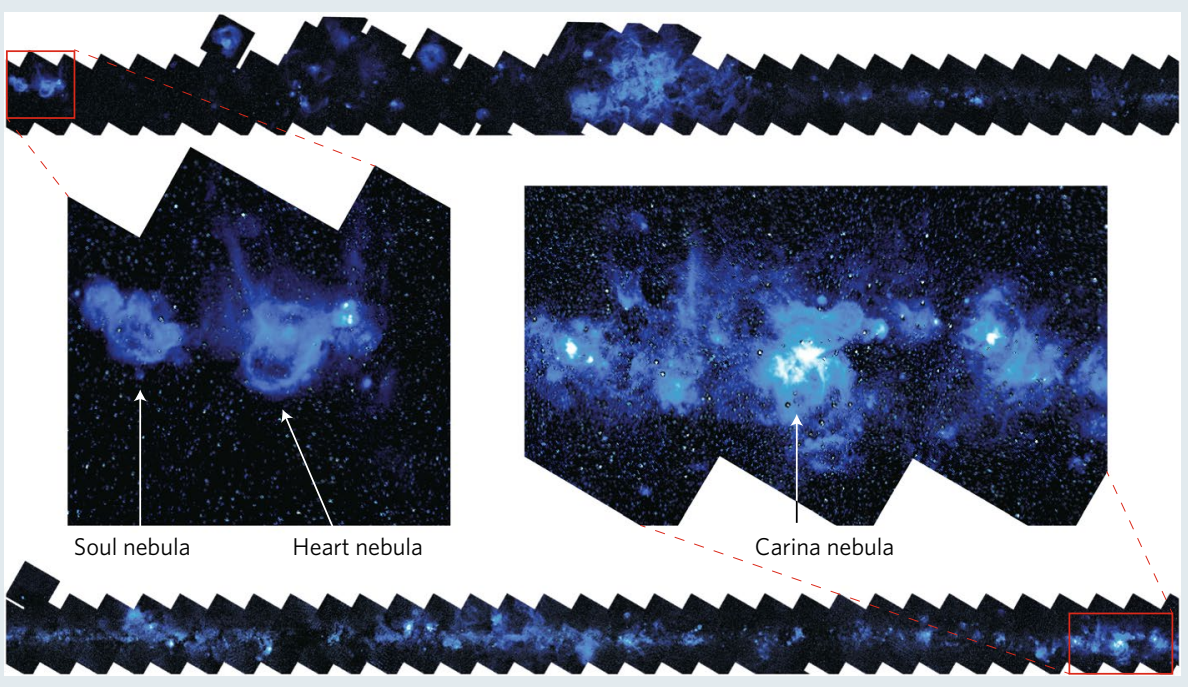

Credit: Images courtesy of Miris Collaboration and resolution (52 arcseconds), combined with the full coverage of the Galactic Plane, are estimated to yield over a 1,000 newly identified $\mathrm{H}$ II regions. Comparison with surveys at shorter wavelengths will also provide a detailed map of dust extinction along the plane.

In addition to the spectroscopic survey of the warm ISM in the Galactic Plane, MIRIS conducted a continuum survey of the north ecliptic pole and the north and south Galactic poles, and a bi-daily continuum monitoring survey towards the north ecliptic pole. The analysis of the continuum survey products will mainly focus on constraining the near-infrared background that is thought to contain signatures of relic emission from high redshift galaxies and accreting primordial black holes at or near the epoch of reionization.

The full dataset has now been released to the public and it can be found on the official MIRIS website (http://miris. kasi.re.kr/miris/) together with more information on the instrument, surveys specifications and data analysis.
Published online: 3 October 2017 DOI: 10.1038/s41550-017-0282-2 